\title{
Binary cuckoo search based optimal PMU placement scheme for united Indian grid - A case study
}

\author{
N. V. Phanendra Babu ${ }^{1 *}$, P. Suresh Babu ${ }^{2}$, D.V. S. S. Sivasarma ${ }^{3}$ \\ ${ }^{1,2,3}$ Department of Electrical Engineering, National Institute of Technology Warangal, INDIA \\ "Corresponding Author: e-mail: phanendra229@gmail.com, Tel +91-7702578106
}

\begin{abstract}
The Phasor Measurement Unit (PMU) is becoming very effective device for power system as it produces synchronized parameters of power system. This measurement data helps us in monitoring, controlling and protecting the system effectively. To design a wide-area measurement system that could observe the complete system, a Binary Cuckoo Search (BCS) method is suggested in this paper. It also considers the system observability under line/PMU outage conditions. After testing the methodology on some benchmark systems, it has been applied on the Indian power grid. The results are then compared with the methods available in literature. This comparison has proven the proposed method as the best and accurate.
\end{abstract}

Keywords: Optimal PMU placement (OPP); Binary Cuckoo Search (BCS); Power system observability

DOI: http://dx.doi.org/10.4314/ijest.v10i2.2

\section{Introduction}

Phasor measurement unit (PMU) is becoming a most important tool for the power system monitoring, control and protection. Some of the applications of PMU (Singh et al., 2011; Babu et al., 2015) include Fault Location, Supervisory Control of Back-Up Zone Protection, Adaptive Out-of-step Relaying coherent group detection, Intelligent islanding, Adaptive Loss-Of-Field Relaying, Adaptive system restoration, Adaptive dependability and security, Generation-load mismatch detection, Identification of generator trip locations, some more applications in the sense of power system monitoring and controlling. So, they are required to be employed for the present and future power systems. So, when a new state estimator commissioned, or an existing estimator is upgraded, the problems of minimizing the number of PMUs and their optimal location for system complete observability will come into picture. An optimal PMU placement (OPP) is quite important during planning studies for both existing and future power networks.

The actual optimization problem was developed in Baldwin et al. (1993). Later, papers (Abur and Magnago, 1999; Magnago and Abur, 2000) suggested OPP in the presence of measurement contingencies for conventional measurement devices which are expensive. After, heuristic algorithms like Genetic Algorithm (Marın et al., 2003) to find the minimal set of PMUs, Particle Swarm Optimization (Hajian et al, 2007; Ahmadi et al., 2011) to obtain minimum PMUs using maximized measurement set, integer linear programming (ILP) (Xu and Abur, 2004; Kavasseri and Srinivasan, 2011), and Binary ILP based real-time monitoring of system (Pal et al., 2014), upgraded Binary Harmony search algorithm (Nazari-Heris and Mohammadi-Ivatloo, 2015), Binary Cat Swarm Optimization (Srivastava and Maheswarapu, 2015), Artificial Bee Colony based Multi- objective Optimal Placement of Phasor Measurement Units (Kulanthaisamy et al., 2014) and multistage PMU placement (Roy et al., 2012) are applied to answer OPP problem. But these methods haven't considered line contingencies. Even though these methods such as single contingency based PMU problem (Rakpenthai et al., 2007) and a conventional measurements based method (Gou, 2008) have answered OPP problem, they ignored ZIBs from search space which may leads to an unreliable state estimation under blackout condition. A binary search scheme (Chakrabarti and Kyriakides, 2008), an integer quadratic based PMU placement (Chakrabarti et al., 2009) without including zero-injection effect, an Optimal PMU Placement strategy considering few pre-specified contingencies (Sodhi 
et al., 2009), participation factor-based method (Caro et al., 2012) are suggested, but none of them have considered all possible contingencies. Recently, authors (Gopakumar et al., 2013a; Gopakumar et al., 2013b; Kumar et al., 2014) have suggested OPP with measurement redundancies without considering channel limits. But the proposed paper considers all possible line/PMU contingencies, channel limits and also zero-injection buses which cannot be ignored from placement for a robust measurement system. This paper suggests a meta-heuristic technique called Binary Cuckoo Search (BCS) for the OPP problem presented in (Babu et al., 2015). The proposed method, after testing on IEEE test systems, is applied to United Indian Grid (UIG).

This paper has been organized as follows. Section 2 gives the problem formulation with constraints considered. Section 3 introduces Binary Cuckoo Search (BCS). Section 4 discusses the results. Finally, section 5 concludes the paper.

\section{PMU Placement Formulation}

Since the optimal PMU placement is an optimization problem, it should be defined with an objective function (1) and some particular constraints. So, the problem will defines as,

Minimize:

$$
\sum_{q \in N} x_{q}
$$

Subjected to:

$$
S_{p} \geq 1, \quad \forall p \in N
$$

Where,

$$
\begin{array}{r}
S_{p}=\sum_{q \in N} C_{p q} x_{q}, \forall p \in N \\
C_{p q}=\left\{\begin{array}{l}
1, \text { if } \mathrm{p}=\mathrm{q} \\
1, \text { if buses } \mathrm{p}, \mathrm{q} \text { are connected } \\
0, \text { otherwise }
\end{array}\right.
\end{array}
$$

If $S_{p}$ is unity or greater than it, then the $p^{\text {th }}$ bus and all its connected buses are observed. This problem will work if and only if there are no abnormal conditions like line outage (fault) or PMU failure (communication channel failure). So, in order to consider these situations which are more probabilistic to occur in power system, it formulates a new approach for observing the system completely. Here, the abnormal conditions will be taken into consideration by modelling each condition as a constraint. The modelled constraints are presented below.

\subsection{Line outage constraint}

This section considers only line outage as the only abnormal condition that would occur on the power system. In this proposed method, the line outage constraint Equation (5) is considered by rewriting the Equation (2) as:

$$
S_{p}^{l} \geq 1, \quad \forall p \in N, \quad \forall l \in L
$$

where

$$
S_{p}^{l}=\sum_{q \in N} C_{p q}^{l} x_{q}
$$

\subsection{PMU failure constraint}

Here, the measurement failure constraint Equation (7) is considered by rewriting the Equation (2) as:

$$
S_{p} \geq 2, \quad \forall p \in N
$$

Where $S_{P}$ is the observability function at $p$ obtained from Equation (3).

\section{3. line/PMU outage constraint}

This part combines the above two equations to achieve the observability under either of these two constraints. Hence, the constraint becomes:

$$
S_{p}^{l}+S_{p} \geq 2, \forall p \in N, \quad \forall l \in L
$$

Throughout the discussion, this paper considers only this condition Equation (8) as the abnormal condition as it combines both line and PMU failure constraints.

\section{Proposed Binary Cuckoo Search (BCS)}

The peculiar behavior of the cuckoo bird is it lays eggs in another bird's nest to let that bird to hatch eggs (Yang, 2009a,b). Then it follows different strategies to minimize the chances of destroying eggs by the host bird. This strategy and behavior has led to the 
introduction of a new naturally-inspired optimization algorithm called Cuckoo Search (CS) algorithm. This Meta-heuristic algorithm was invented by Xin-SheYang and Suash Deb in the year of 2009. From the basic cuckoo search algorithm, each cuckoo uses Levy's flights to search a new nest for laying its egg. Levy flight is a model walk function to direct a cuckoo towards a new nest, which is random and characterized by some pre-defined step lengths for obeying power-law distribution. These levy flights are very helpful in optimizing any problems of engineering and sciences. According to standard CS, every cuckoo will searches for nest on ensuring the following rules ideally.

Rule 1: Each cuckoo should lay only one egg at a time, and choose nest randomly.

Rule 2: The best nest with an egg of most and highest fitness should be passed on to the next generation.

Rule 3: The number of host nests should be fixed, and the probability $\left(\mathrm{p}_{\mathrm{a}}\right)$ with which the host bird can discover the egg laid by the cuckoo should lies in the range of $[0,1]$, i.e. $\mathrm{p}_{\mathrm{a}} \in[0,1]$.

The equations (9) and (10), given below, together help in generating new solution using levy flights with their random steps from the levy distribution function (Yang and Deb, 2010). Moreover the random walk process helps cuckoo in its consecutive jumping by obeying power-law step-length distribution with a heavy tail.

$$
\begin{gathered}
x_{i}^{t+1}=x_{i}^{t}+\alpha \oplus \operatorname{levy}(\lambda) \\
\text { levy } \sim u=t^{-\lambda}
\end{gathered}
$$

Here, $x_{i}^{t}$ and $x_{i}^{t+1}$ are the solutions at instants $t$ and $t+1$. Here, $\alpha$ represents step size whose value varies according to the scale of the problem of interest. The operator $\oplus$ does the entry-wise multiplications. Even though this operator is as similar as that of PSO, it is more efficient than that of PSO.

The present OPP problem is a binary optimization problem whose solution is a set of zeros (0s) and ones (1s). Here, binary one represents the presence of PMU and binary zero represents the absence of PMU. But, the search space for the standard CS is continuous which means its solution is a set of real number. Hence, it cannot be applied for the proposed binary optimization problem whose solution should be a binary solution. So, a new version, Binary Cuckoo Search is introduced here.

\subsection{Binary Cuckoo Search (BCS)}

Essentially the BCS contains two functional blocks. First block contains two cuckoo dynamic operations namely Levy flights and binary solution representation (BSR). Here, Levy flight will be used to search a new cuckoo (Ouaarab et al., 2014; Gherboudj et al., 2012). As the main intension of applying BCS is to handle binary optimization problems efficiently, the BCS has to transform a real valued solution $\left(\mathrm{x}_{\mathrm{r}}\right)$ to binary value $\left(\mathrm{x}_{\mathrm{b}}\right)$. This can be done with the help of sigmoid function given in Equation (11). Sigmoid function will calculates the flipping chances of each cuckoo. Later, these flipping chances will be used to compute the binary value of that particular cuckoo. This transformation can be done as follows.

$$
\sigma(x r)=\frac{1}{\left(1+e^{-x_{r}}\right)}
$$

where $\sigma\left(x_{r}\right)$ represents the flipping chance of bit ' $x_{b}$ '. After getting $\sigma\left(x_{r}\right)$, we will compare it with a randomly generated number $\gamma$, where $\gamma \in[0,1]$ for each dimensions of ' $r$ '. Then the transformation Equation (12) will be done as follows:

$$
x_{b}=\left\{\begin{array}{l}
1, \text { if } \gamma<\sigma\left(x_{r}\right) \\
0, \text { otherwise }
\end{array}\right.
$$

As shown in Figure 1, the two operators (Levy flights and BSR) will combine the basic CS algorithm with sigmoid function to achieve BCS. The second functioning block has selection operator and objective function. Here, the selection operation is nothing but the elitism phenomenon applied in genetic algorithm.

\section{Algorithm:}

Step 1: Read $\mathrm{P}_{\mathrm{a}}$ and objective function

Step 2: Initialize the population of $\mathrm{N}$ host nests

Step 3: While ( $\mathrm{t}<$ maximum generation) or (convergence criterion), repeat the steps 4 to 12.Otherwise, stop the procedure.

Step 4: Obtain a cuckoo (let i) randomly using Levy flights

Step 5: Obtain its binary representation by BSR algorithm and calculate its fitness $S_{\mathrm{i}}$.

Step 6: Select a nest from $\mathrm{N}$ (let j) arbitrarily

Step 7: Obtain its binary representation by BSR algorithm and calculate its fitness $S_{j}$.

Step 8: If $\left(\mathrm{S}_{\mathrm{i}}>\mathrm{S}_{\mathrm{j}}\right)$, replace $\mathrm{j}$ by the new solution and go to next step9, else go directly to step9.

Step 9: Obtain the binary representation from the BSR algorithm for all nests and, evaluate their fatnesses.

Step 10: Abandon a fraction $\left(\mathrm{P}_{\mathrm{a}}\right)$ of worse nests. Build new nests at new locations via Levy flights.

Step 11: Obtain the binary representation there, using BSR algorithm and, calculate their fitness.

Step 12: Keep the nests with highest fitness and order the solutions in descending order of their fitness and find the latest best. 


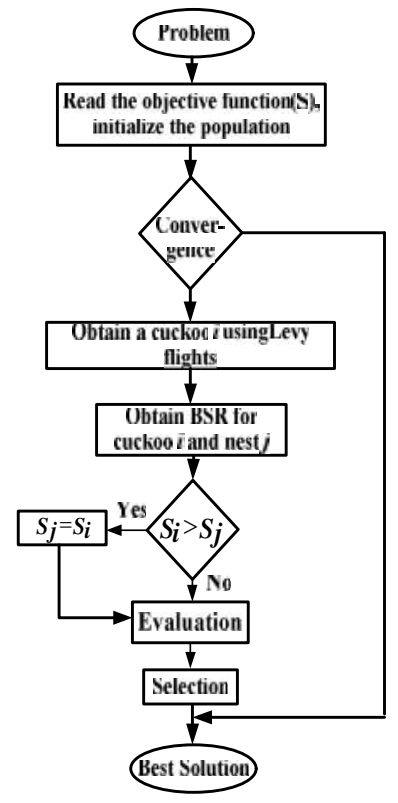

Figure 1. Functional diagram of BCS

\section{Results and discussions}

This methodology, after testing it on few IEEE test systems, has been applied on different Inter Regional power Grids (IRGs) (Power Grid 2018). It is also applied on Unite Indian Grid (UIG). The results that obtained are tabulated in Tables 1and 2. The results are compared with some of the methods from literature and the comparison is presented in Table 3 and Table 4. The results show that, unlike the methods discussed in literature, the proposed method have identified the number PMUs required for observing the system under both normal and line/PMU failure outage conditions respectively.

Table 1. PMU locations under normal conditions

\begin{tabular}{|l|l|l|}
\hline system & Location of PMUs & $\begin{array}{l}\text { No. } \\
\text { PMUs }\end{array}$ \\
\hline IEEE-9 & $4,6,8$ & 3 \\
IEEE-14 & $2,6,8,9$ & 4 \\
IEEE-30 & $2,4,6,10,11,12,19,24,26,29$ & 10 \\
IEEE-57 & $1,4,9,20,24,27,29,30,32,36,38,39,41,45,46,51,54$ & 17 \\
IEEE-118 & $2,5,9,12,13,17,21,23,26,29,34,37,42,45,49,53,56,62,64,71,75,77,80,85,86,90,94,10$ & 32 \\
& $1,105,110,115,116$ & \\
IEEE-300 & $2,3,5,10,13,15,23,24,26,33,36,42,43,51,55,59,60,63,70,71,73,74,77,84,86,97,102,1$ & 87 \\
& $03,104,107,108,109,114,119,122,124,130,132,133,134,137,139,140,144,145,152,1$ & \\
& $54,159,161,162,166,173,178,181,184,189,193,194,200,204,206,211,214,215,219,2$ & \\
& $21,225,230,231,232,234,237,238,240,244,245,249,9012,9002,9022,9023,9003,900$ & \\
& $4,9005,9533,9007,531$. & \\
\hline
\end{tabular}


Table 2. PMU locations under line/PMU outage

\begin{tabular}{|c|c|c|}
\hline system & Location of PMUs & $\begin{array}{l}\text { No. of } \\
\text { PMUs }\end{array}$ \\
\hline IEEE-9 & $1,2,3,4,6,8$ & 6 \\
\hline IEEE-14 & $1,2,3,6,7,8,9,11,13$ & 9 \\
\hline IEEE-30 & $1,2,4,6,7,8,9,10,11,12,13,15,17,18,19,21,24,25,26,29,30$ & 21 \\
\hline IEEE-57 & $\begin{array}{l}1,2,4,6,9,11,12,15,19,20,22,24,25,26,28,29,30,32,33,35,36,38,39,41,45,46,47,50,51,53 \text {, } \\
54,56,57\end{array}$ & 33 \\
\hline IEEE-118 & $\begin{array}{l}1,3,5,7,9,10,11,12,15,17,19,21,22,24,26,27,28,30,31,32,34,36,37,40,42,44,45,46,49,50, \\
52,53,56,58,59,62,63,64,66,68,71,73,74,75,77,78,80,84,85,86,87,89,90,92,94,96,100,10 \\
1,105,107,108,110,111,112,114,116,117,118\end{array}$ & 68 \\
\hline IEEE-300 & $\begin{array}{l}2,3,5,7,11,12,13,14,15,16,17,20,23,24,26,33,35,36,39,41,42,43,44,48,51,52,54,58,59,60 \\
, 62,63,69,70,71,72,74,77,78,81,84,85,86,87,89,90,94,98,100,103,104,105,108,109,110, \\
113,114,116,117,118,119,121,122,124,126,130,132,133,135,136,137,138,140,142,143, \\
145,147,153,155,156,157,160,162,164,166,167,168,170,171,172,174,175,177,178,182, \\
183,184,185,187,188,191,194,197,198,200,201,205,206,208,209,210,211,213,214,215, \\
217,219,221,222,223,225,226,227,229,230,231,232,233,234,236,237,238,239,240,241, \\
244,245,246,249,250,281,9012,9002,9021,9022,9023,9024,9025,9026,9003,9031,9032, \\
9033,9034,9035,9036,9037,9038,9004,9041,9042,9043,9005,9051,9052,9053,9054,905 \\
5,9533,9007,9071,9072,9121,319,320,526,528,7071,531,562,552,609,323,322,324 .\end{array}$ & 185 \\
\hline
\end{tabular}

Table 3. Comparison of the PMU number considering normal condition

\begin{tabular}{|l|l|l|l|l|l|l|}
\hline Optimization Method & IEEE-9 & IEEE-14 & IEEE-30 & IEEE-57 & IEEE-118 & IEEE-300 \\
\hline BCS & 3 & 4 & 10 & 17 & 32 & 87 \\
\hline $\begin{array}{l}\text { Kulanthaisamy et al. } \\
\text { (2014) }\end{array}$ & N/A & 4 & 10 & 17 & 32 & N/A \\
\hline Roy et al. (2012) & N/A & 4 & 10 & 17 & 32 & N/A \\
\hline Ahmadi et al. (2011) & N/A & 4 & N/A & 17 & 32 & N/A \\
\hline $\begin{array}{l}\text { Kavasseri and } \\
\text { Srinivasan (2011) }\end{array}$ & N/A & 4 & N/A & N/A & 32 & N/A \\
\hline Pal et al. (2014) & N/A & N/A & N/A & N/A & N/A & 87 \\
\hline
\end{tabular}

N/A-Not Applied

Table 4. Comparison of the PMU number considering line/PMU failure

\begin{tabular}{|c|c|c|c|c|c|c|}
\hline Optimization Method & IEEE-9 & IEEE-14 & IEEE-30 & IEEE-57 & IEEE-118 & IEEE-300 \\
\hline BCS & 6 & 9 & 21 & 33 & 68 & 185 \\
\hline $\begin{array}{l}\text { Kulanthaisamy et al. } \\
\text { (2014) }\end{array}$ & N/A & N/A & N/A & N/A & N/A & N/A \\
\hline Roy et al. (2012) & N/A & N/A & N/A & N/A & N/A & N/A \\
\hline Ahmadi et al. (2011) & N/A & N/A & N/A & N/A & N/A & N/A \\
\hline $\begin{array}{ll}\text { Kavasseri } & \text { and } \\
\text { Srinivasan (2011) } & \end{array}$ & N/A & N/A & N/A & N/A & N/A & N/A \\
\hline Pal et al. (2014) & N/A & N/A & N/A & N/A & N/A & N/A \\
\hline
\end{tabular}

4.1. Regional Indian power Grids

Indian power Grid has five Inter Regional power Grids (IRGs), such as Northern Region of Indian power Grid (NRIG), Southern RIG (SRIG), Eastern RIG (ERIG), Western RIG (WRIG) and North-Eastern RIG (NERIG). This article studies the PMU placement for the above five Regional Indian power Grids.

\subsection{Western Regional Indian power Grid (WRIG)}

WRIG has four states (Chhattisgarh, Maharashtra, Madhya Pradesh and Gujarat) with 77 buses. The number and the locations of PMUs required for observing WRIG completely are given in the Table 5. The PMUs are also located graphically as shown in Figure 2 and Figure 3. The results say that WRIG requires 19 and 44 PMUs to observe the system under the normal and line/PMU failure conditions respectively. It is to be noted that the PMU locations are numbered as per the numbers given for buses. 
Table 5. PMU locations for WRIG without channel limits

\begin{tabular}{|c|c|c|c|c|}
\hline \multirow[t]{2}{*}{ System } & \multicolumn{2}{|c|}{ Under normal operating conditions } & \multicolumn{2}{|l|}{ under line/PMU outage conditions } \\
\hline & PMU locations & $\begin{array}{l}\text { No. of } \\
\text { PMUs }\end{array}$ & PMU locations & $\begin{array}{l}\text { No. of } \\
\text { PMUs }\end{array}$ \\
\hline WRIG & $\begin{array}{l}418,421,425,429,433,4 \\
39,440,443,448,456,46 \\
0,464,466,471,474,480, \\
485,488,491 .\end{array}$ & 19 & $\begin{array}{l}418,420,421,423,425,426,427,431 \\
, 432,433,437,439,441,443,445,44 \\
6,464,466,467,468,469,470,47144 \\
8,449,451,454,455,457,460,461,4 \\
72,473,474,475,477,478,480,484, \\
486,487,488,489,492\end{array}$ & 44 \\
\hline
\end{tabular}

4.3. Eastern Region Indian power Grid (ERIG)

ERIG has five states with 90 buses. It is the only IRG to be having connectivity with all the remaining IRGs. The ERIG requires 24 and 57 PMUs to observe the system under normal and abnormal conditions respectively. The locations of PMUs required for observing the WRIG completely are tabulated Table 6 and are represented graphically in Figure 4 and Figure 5.

Table 6. PMU locations for ERIG without channel limits

\begin{tabular}{|l|c|c|c|c|}
\hline System & $\begin{array}{c}\text { under normal operating } \\
\text { conditions }\end{array}$ & \multicolumn{2}{|c|}{ under line/PMU outage conditions } \\
\cline { 2 - 5 } & PMU locations & $\begin{array}{c}\text { No. of } \\
\text { PMUs }\end{array}$ & PMU locations & $\begin{array}{c}\text { No. of } \\
\text { PMUs }\end{array}$ \\
\hline ERIG & $314,317,319,321$, & 24 & $314,315,316,317,319,320,321,322,3$ & 57 \\
& $326,329,330,338$, & & $24,326,327,329,331,332,334,337,33$ & \\
& $339,345,351,352$, & & $8,339,340,341,343,345,346,348,351$ & \\
& $354,360,367,371$, & & $352,353,354,356,360,361,362,364$, & \\
& $374,376,378,385$, & & $367,369,370,373,374,375,376,377,3$ & \\
& $388,391,396,401$. & & $78,380,381,384,385,388,389,391,39$ & \\
& & & $2,395,396,397,398,399,401,402$. & \\
\hline
\end{tabular}

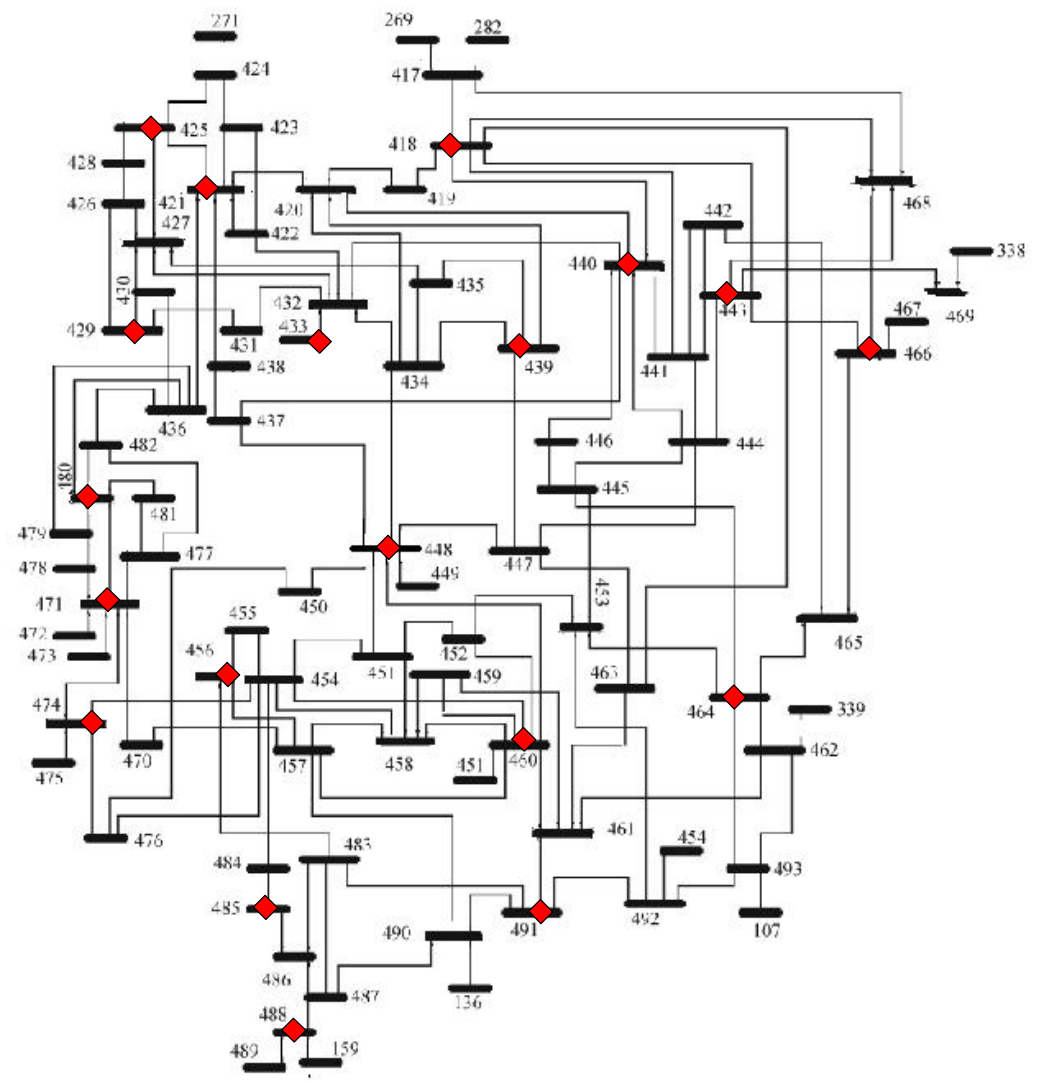

Figure 2. PMU locations in WRIG under normal operating conditions 


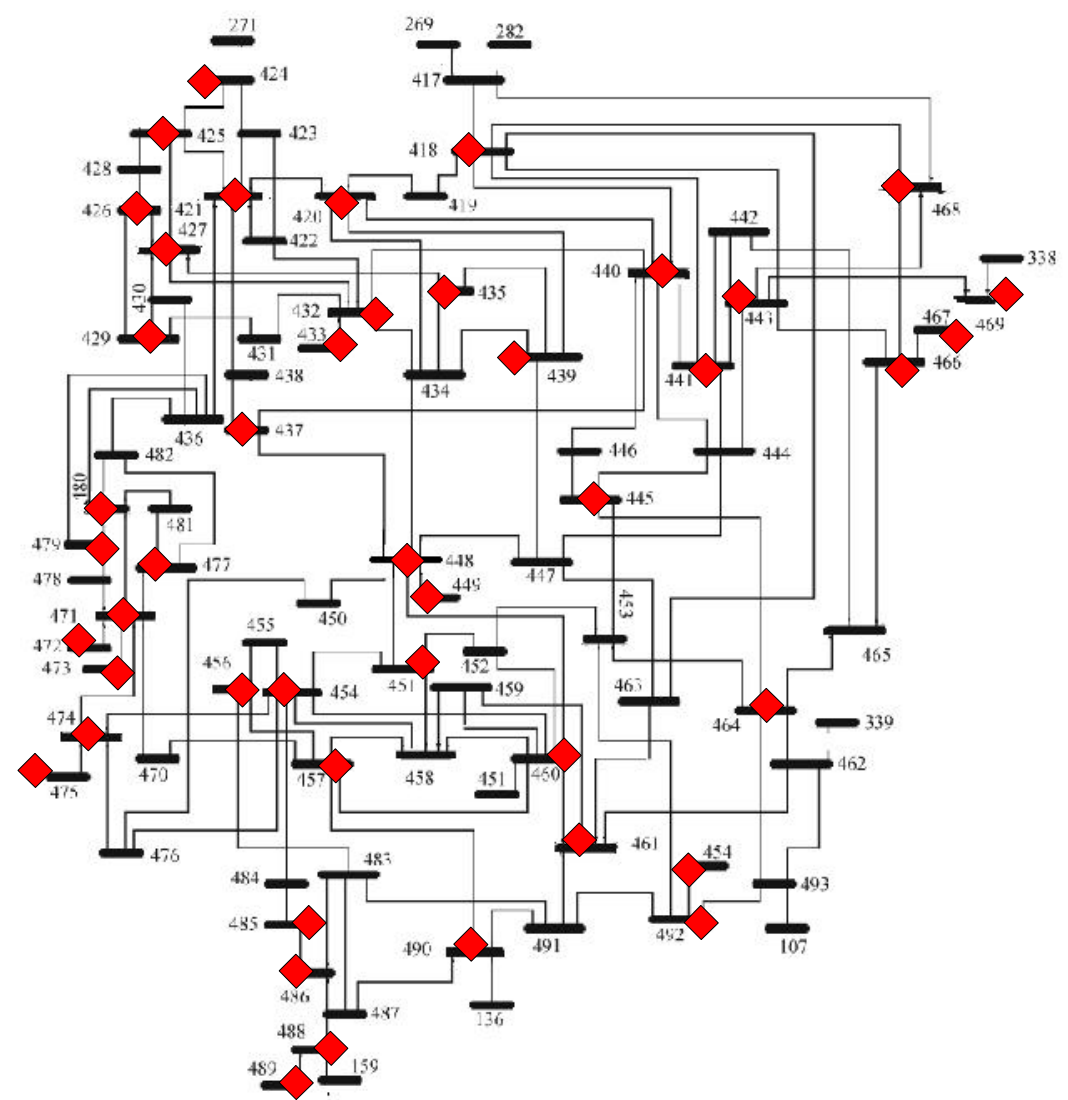

Figure 3. PMU locations in WRIG under line outage/ PMU failure conditions.

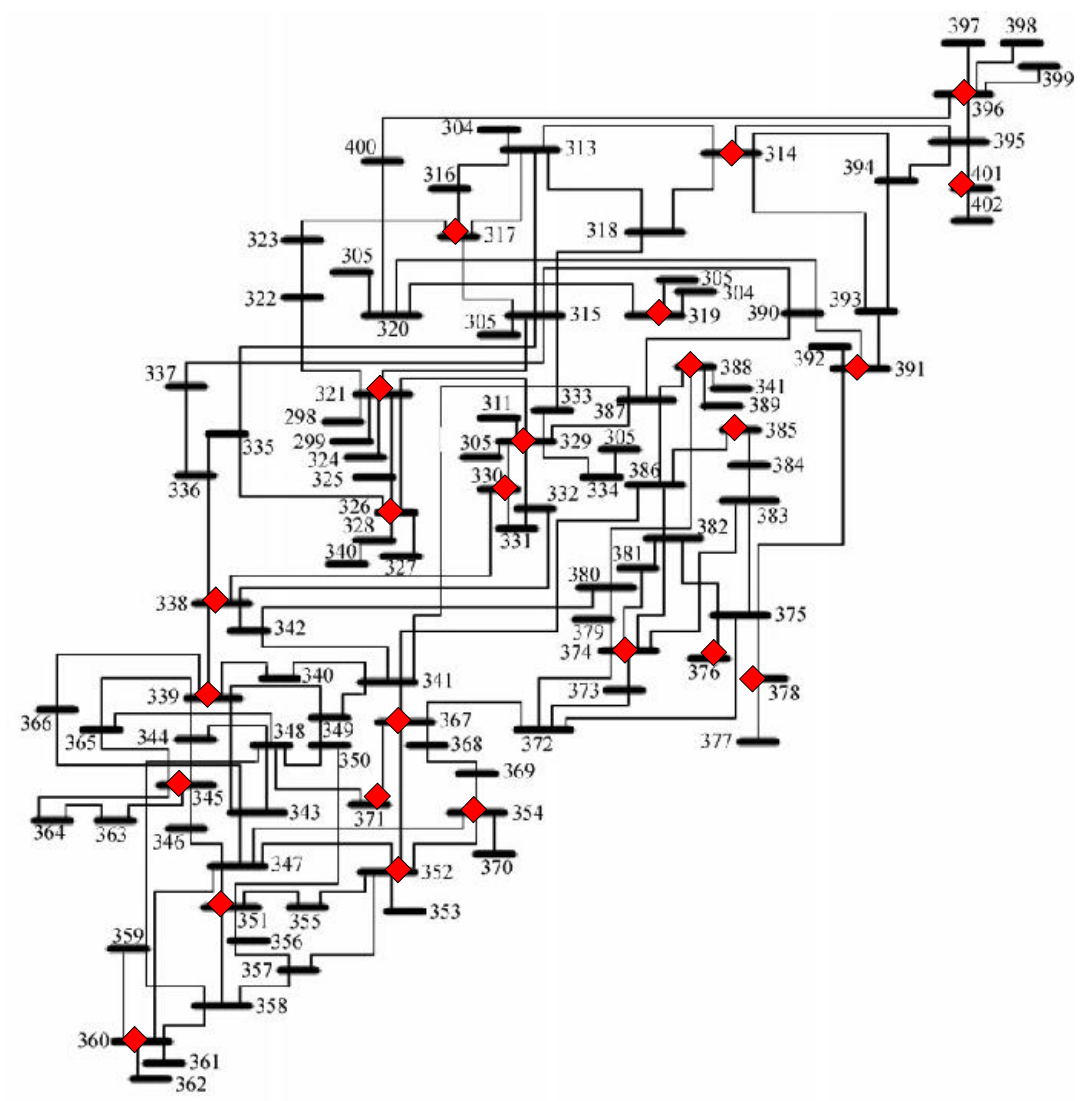

Figure 4. PMU locations in ERIG under normal operating conditions 


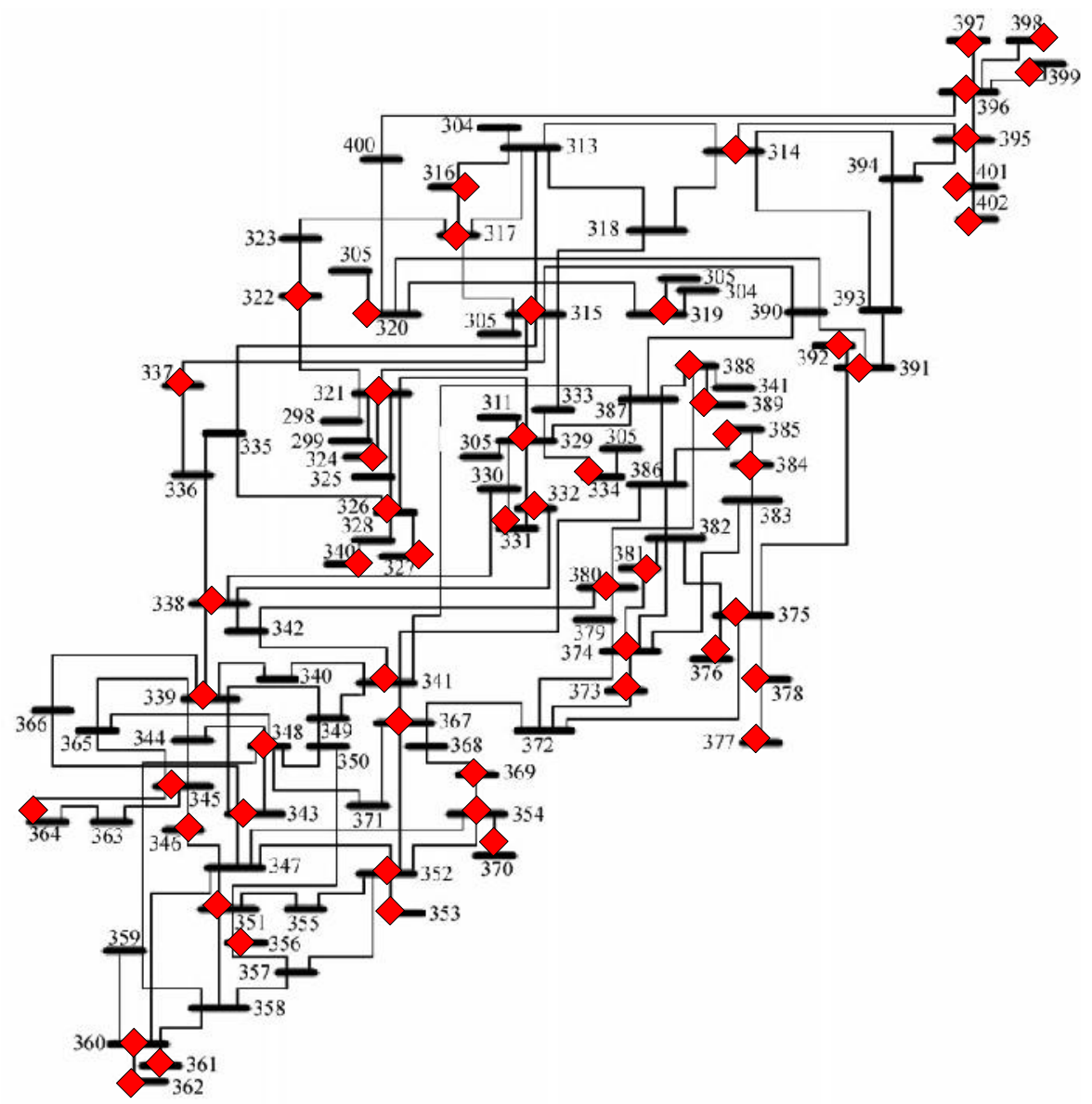

Figure 5. PMU locations in ERIG under line outage/ PMU failure conditions

\subsection{Northern Regional Indian power Grid (NRIG)}

NRIG is the biggest RIG with 104 buses that covers almost 30\% of the India's geographical area, among the all five regions. It has totally seven states and two union territories. From the results obtained, as shown in Table 7, it is proven that, to acheive complete observability, NRIG requires 27 and 65 PMUs to observe the system under normal and abnormal conditions respectively. The locations of PMUs are clearly mentioned Figure 6 and Figure 7.

Table 7. PMU locations for NRIG without channel limits

\begin{tabular}{|l|c|c|c|c|}
\hline \multirow{2}{*}{ System } & \multicolumn{2}{|c|}{ under normal operating conditions } & \multicolumn{2}{c|}{ under line/PMU outage conditions } \\
\cline { 2 - 5 } & PMU locations & No. of PMUs & PMU locations & No. of PMUs \\
\hline NRIG & $210,212,216,218,22$ & 27 & $209,210,211,212,213,215,216,2$ & 65 \\
& $4,228,231,232,236,2$ & & $17,219,220,221,222,224,225,22$ & \\
& $38,242,249,250,254$, & & $6,228,229,231,233,235,236,237$ & \\
& $255,259,262,265,26$ & & $, 238,241,242,244,245,247,249$, & \\
& $9,271,277,281,286,2$ & & $250,252,254,255,258,259,262,2$ & \\
& $96,301,305,308,309$. & & $63,265,266,267,269,271,272,27$ & \\
& & & $3,275,277,278,279,280,281,282$ & \\
& & & $, 284,285,286,288,289,293,295$, & \\
& & & $296,297,299,301,303,305,306,3$ & \\
\hline
\end{tabular}




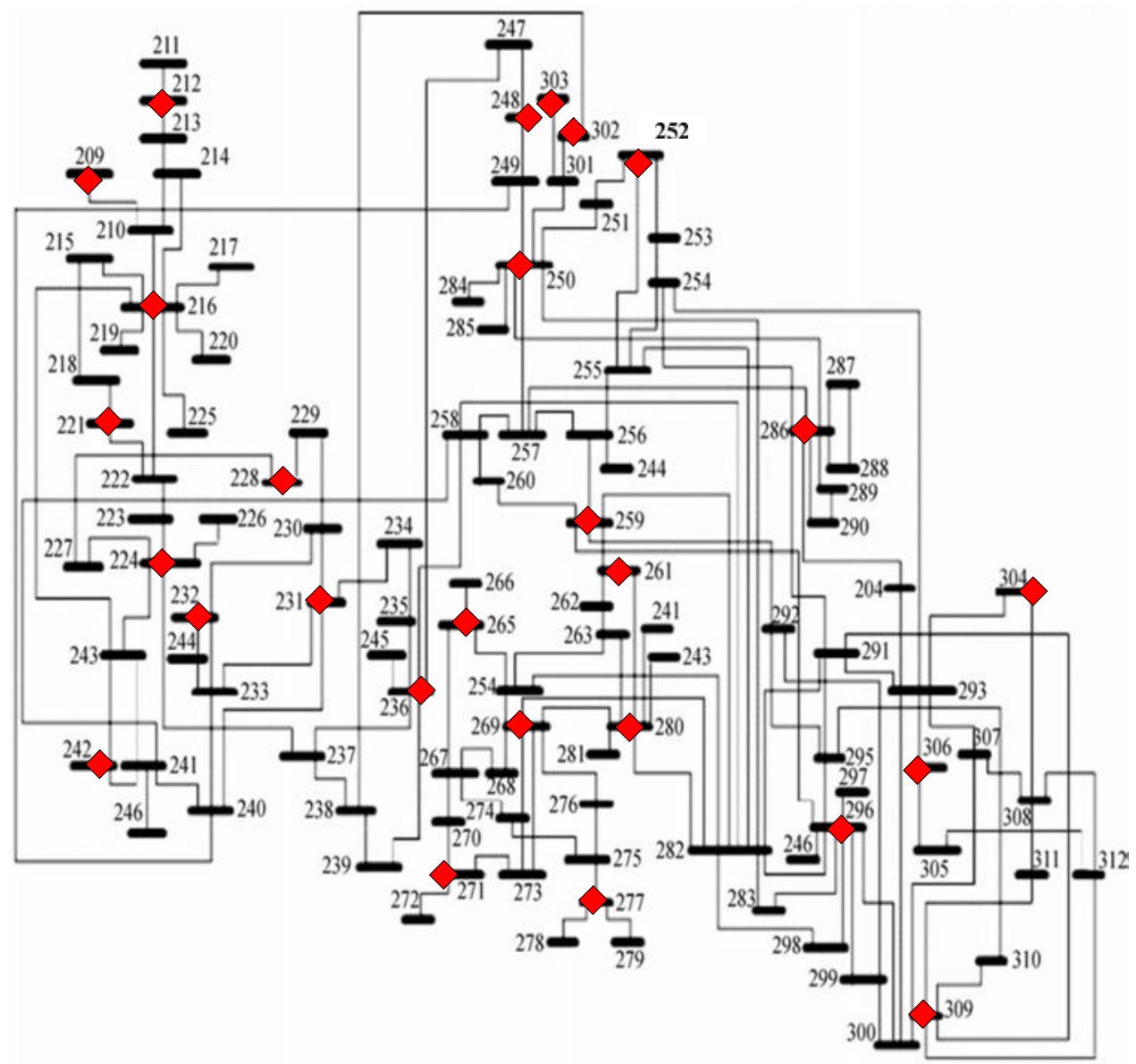

Figure 6. PMU locations in NRIG under normal operating conditions

\subsection{Southern Regional Indian power Grid (SRIG)}

SRIG has 208 buses with four states. This is the only IRG that was connected asynchronously to the remaining parts of the Indian power grid till December 31, 2012, after which it was synchronized to the rest of Indian power grid to form a single Indian grid. From the results, without considering channel limits, it requires 56, 132 PMUs to observe the network under normal and abnormal conditions respectively. The results are given in Table 8 and are also represented with the help of Figure 8 and Figure 9.

Table 8. PMU locations for SRIG

\begin{tabular}{|l|l|c|c|c|}
\hline System & \multicolumn{2}{|c|}{ under normal operating conditions } & \multicolumn{2}{|c|}{ under line/PMU outage conditions } \\
\hline & PMU locations & $\begin{array}{c}\text { No. of } \\
\text { PMUs }\end{array}$ & PMU locations & $\begin{array}{c}\text { No. of } \\
\text { PMUs }\end{array}$ \\
\hline SRIG & $5,7,10,12,19,21,27,30,33,35$, & 56 & $1,3,4,6,9,10,12,13,14,16,19,22,23,25,26,27,28$ & 132 \\
& $47,48,49,54,58,60,63,67,73$, & & $, 30,31,32,33,35,36,37,42,43,45,47,48,49,54,5$ & \\
& $75,83,88,89,92,95,99,103,10$ & & $6,57,58,59,60,62,63,64,66,67,69,70,71,73,75$, & \\
& $7,110,111,115,117,118,121$, & & $76,78,79,83,85,86,87,88,89,92,93,95,96,98,99$ & \\
& $122,127,128,132,134,136,13$ & & $, 100,103,104,106,107,108,110,111,112,113,1$ & \\
& $8,145,147,152,154,157,159$, & & $15,116,117,118,119,120,121,122,123,124,127$ & \\
& $162,165,185,188,190,192,19$ & & $128,130,132,133,134,136,138,141,142,145,1$ & \\
& $6,201,208$ & & $46,147,148,150,152,154,157,158,159,160,161$ & \\
& & & $, 163,164,165,166,167,169,170,174,175,177,1$ & \\
& & & $79,180,181,184,185,188,190,191,192,194,196$ & \\
& & & $197,198,201,202,203,205,207,208$. & \\
\hline
\end{tabular}




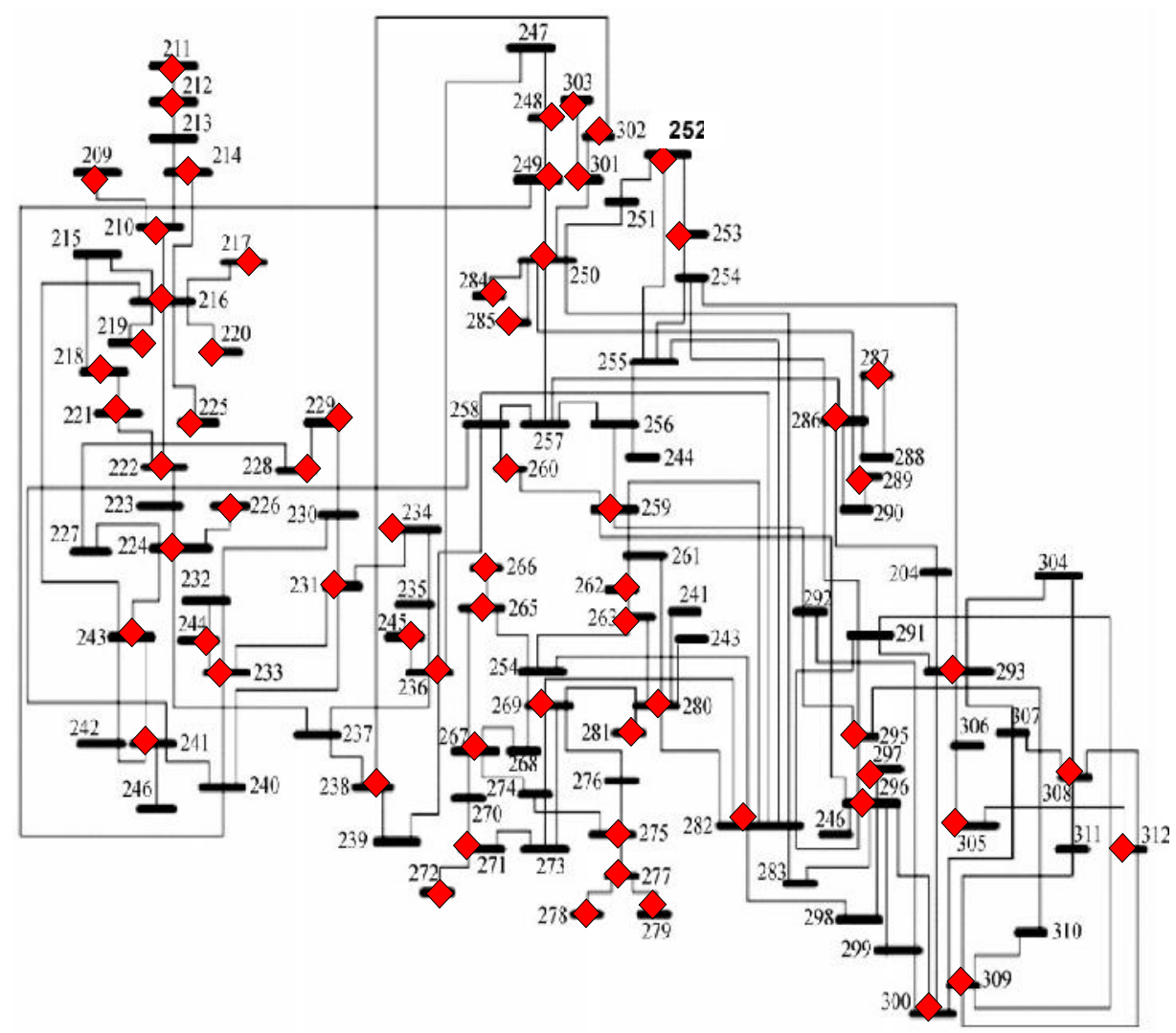

Figure 7. PMU locations in NRIG under line outage/ PMU failure conditions

4.6. North eastern Regional Indian power Grid (NERIG)

NERIG has seven states with 14 buses. As shown in Table 9, NERIG requires 5, 12 PMUs to observe the network for the two conditions respectively. The locations are clearly depicted in Figures 10, 11.

Table 9. PMU locations for NERIG

\begin{tabular}{|c|c|c|c|c|}
\hline \multirow{2}{*}{ System } & under normal operating conditions & \multicolumn{2}{c|}{ under line/PMU outage conditions } \\
\cline { 2 - 5 } & PMU locations & $\begin{array}{c}\text { No. of } \\
\text { PMUs }\end{array}$ & PMU locations & $\begin{array}{c}\text { No. of } \\
\text { PMUs }\end{array}$ \\
\hline NERIG & $404,406,407,411,413$ & 5 & $403,404,405,406,407,409,410,412,413,414,415,416$ & 12 \\
\hline
\end{tabular}

\subsection{United Indian Grid (UIG)}

After successful synchronization of SRIG to NEW (Northern-Eastern-Western) grid, on December 31st 2013, India became the nation with one grid and one frequency. So the wide area measurement system must be reliable and robust. This section presents an optimal PMU location which can observe the whole Indian Grid even under abnormal conditions like line/PMU outage case, which has considered the new synchronous line connected between SRIG and NEW grid of India. This section also gives the detailed locations of PMUs for Indian Grid under both normal and abnormal conditions on considering channel limitations also. The results are tabulated in Table 10 . 


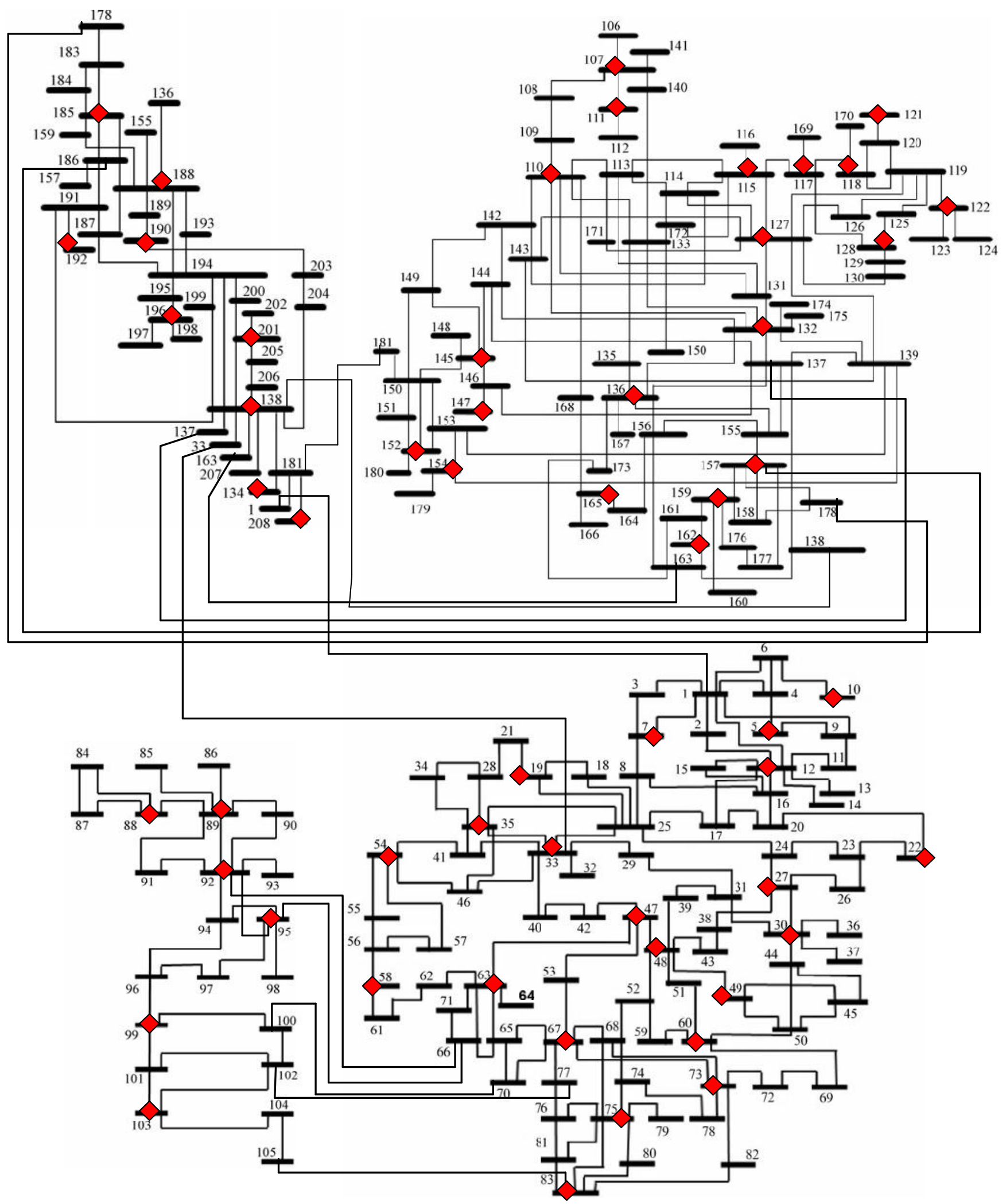

Figure 8. PMU placement for SRIG considering normal condition 


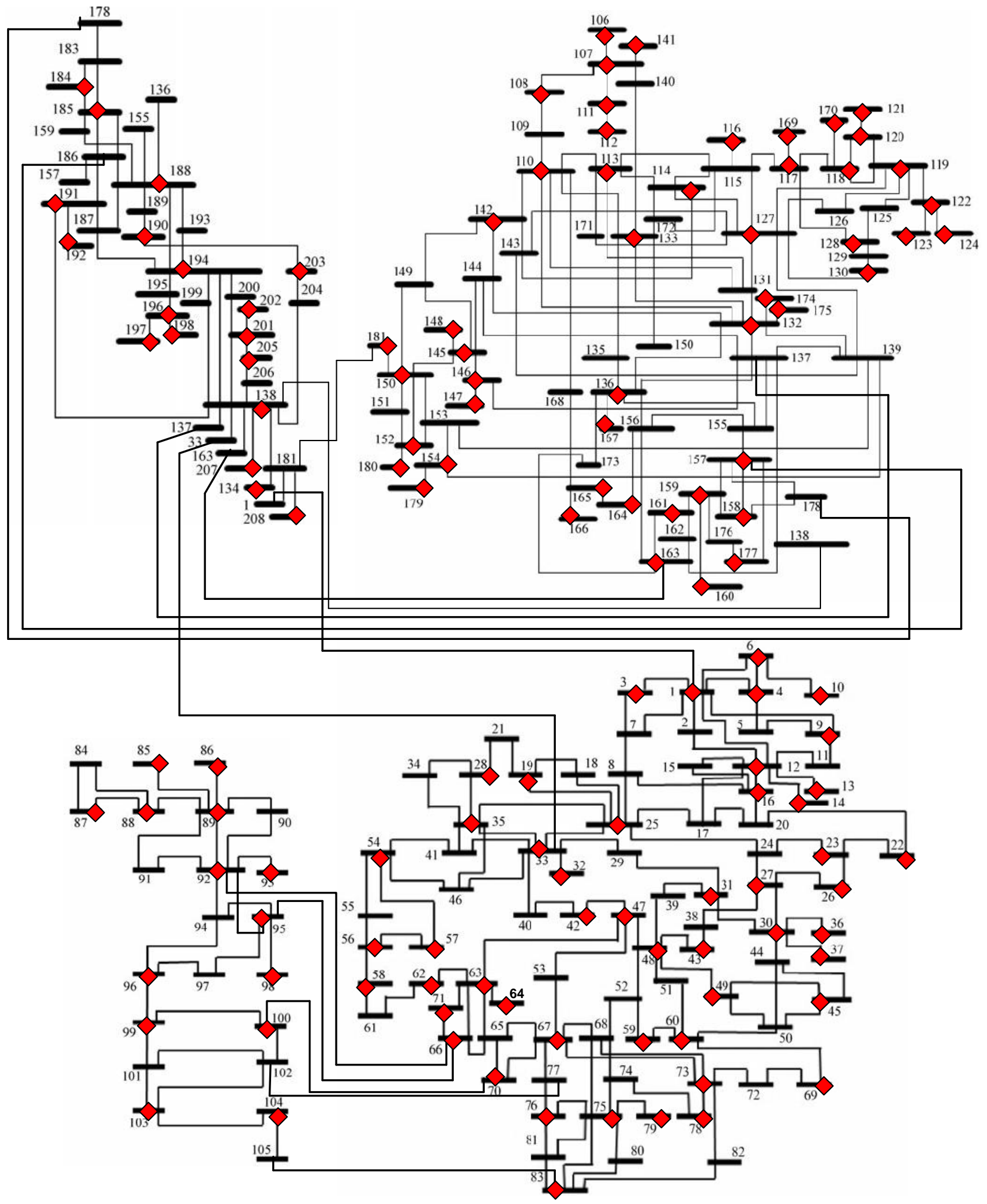

Figure 9. PMU placement for SRIG considering line/PMU outage 


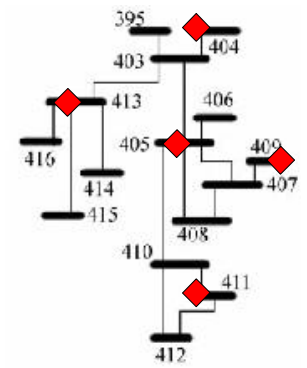

Figure 10. PMU locations in NERIG under normal condition

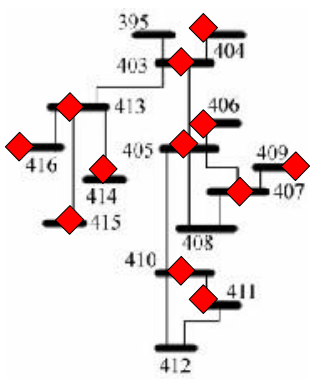

Figure 11. PMU locations in NERIG considering line/PMU outage

Table 10. PMU locations for UIG without channel limits

\begin{tabular}{|c|c|c|}
\hline System & $\begin{array}{l}\text { PMU locations under normal } \\
\text { operating conditions }\end{array}$ & PMU locations under line/PMU outage conditions \\
\hline UIG & $\begin{array}{l}5,6,7,12,19,22,27,30,33,35,47,48,50, \\
55,56,58,60,63,70,73,75,76,84,89,93, \\
95,96,103,105,107,110,111,115,117, \\
118,121,122,126,127,128,132,136,13 \\
8,145,146,152,157,159,163,165,168, \\
169,179,181,185,188,190,191,196,20 \\
1,204,205,209,212,216,221,224,226, \\
229,231,236,239,242,243,244,247,25 \\
0,252,259,262,265,269,271,277,281, \\
286,296,301,304,305,309,311,315,31 \\
7,320,321,326,332,338,339,345,351, \\
352,354,359,360,367,374,375,378,38 \\
1,385,388,391,395,396,401,403,405, \\
409,411,413,420,421,428,431,433,43 \\
5,441,445,447,448,456,460,466,471, \\
474,480,484,486,488,492 . \\
\quad \text { Number of PMUs=142 }\end{array}$ & $\begin{array}{c}1,5,6,7,9,10,12,13,14,15,19,20,22,23,25,27,28,30,31,32,33,35,36, \\
37,38,42,44,45,47,48,54,56,57,58,59,60,61,63,64,67,69,70,71,73, \\
74,75,77,79,81,83,85,86,87,88,89,92,93,95,96,98,99,101,104,105 \\
, 106,107,109,110,111,112,115,116,117,118,119,122,123,124,125 \\
, 127,129,130,132,133,134,136,138,141,142,145,146,147,148,151 \\
, 152,154,157,158,159,160,161,163,164,165,166,167,169,170,171 \\
, 174,175,177,179,180,181,184,185,188,190,191,192,194,196,197 \\
, 198,201,202,203,205,207,208,209,210,211,212,213,216,217,218 \\
, 219,220,221,223,224,225,226,228,229,230,231,232,235,236,238 \\
, 241,243,244,245,248,249,250,252,254,255,259,260,261,262,265 \\
, 266,267,269,271,272,275,277,278,279,280,281,282,284,285,286 \\
, 287,290,292,293,295,296,297,301,302,303,304,305,309,312,314 \\
, 317,318,320,321,322,324,326,327,328,329,330,332,333,336,337 \\
, 338,339,345,346,350,351,352,353,354,356,358,360,361,362,364 \\
, 366,367,368,370,371,373,374,375,376,377,378,380,381,384,385 \\
, 387,388,389,391,392,393,396,397,398,399,401,402,403,404,405 \\
, 406,407,409,410,412,413,414,415,416,419,420,421,423,425,426 \\
, 429,432,433,434,436,438,439,441,443,445,446,448,449,450,452 \\
4454,456,457,460,461,463,465,466,467,471,472,473,474,475,477 \\
, 478,480,483,484,486,488,489,493 . \\
\text { Number of PMUs=312}\end{array}$ \\
\hline
\end{tabular}

4.8. Economies of the proposed algorithm

It presents the net savings befitted from the suggested algorithm. It means, if we consider the cost of one PMU as one unit, the costs incurred for the system complete observability without OPP, with OPP under normal and line/PMU outage conditions would corresponds to the number of PMUs required in the respective conditions. So, WRIG gets benefited with $75.32 \%$ and $42.85 \%$ of savings under the two conditions respectively. Similarly, while deploying the phasor measurement system, ERIG saves $72.22 \%$ and $35.56 \%$, NRIG saves $74.04 \%$ and $37.5 \%$, SRIG saves $73.07 \%$ and $24 \%$, and NERIG saves $64.29 \%$ and $14.29 \%$ respectively. The net savings of different IRGs and UIG are clearly depicted in Figure 12. 


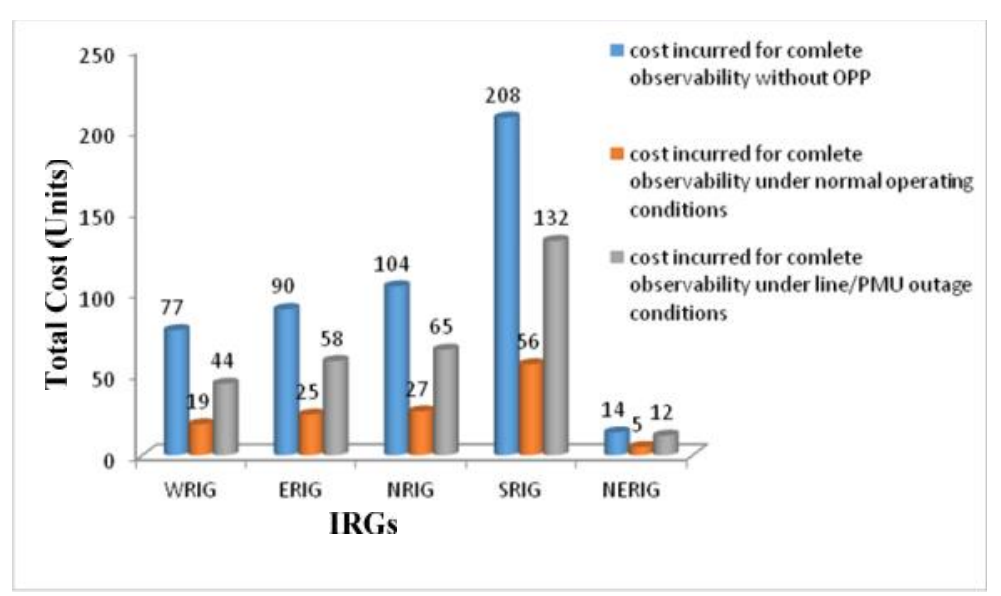

Figure 12. Economies of the proposed algorithm

\section{Conclusion}

As the power system is highly prone to repeated faults or disturbances, it is needed to be monitored continuously. This paper has considered the conditions like line outage and measurement failure for finding PMU placements. This really helps for the both existing and future networks. The proposed BCS method has solved the OPP problem for all types of power systems accurately. The proposed method has been tested on few IEEE standard test systems, and then applied for United Indian power Grid (UIG). From the results and discussions, number of PMUs required for the system complete observability is almost one-third of the system size. And, this is almost doubled if single line/PMU outage were considered. Hence, it is verified that the proposed method has optimized the OPP problem efficiently and benefited economically.

\section{Nomenclature}

$x_{q:}$ objective function

$N$ : Bus set

$C_{p q:}$ Binary connectivity parameter

$C_{p q:}^{l}$ Binary connectivity parameter for $l^{\text {th }}$ line outage

\author{
$S_{p}$ : observability function at bus $\mathrm{p}$ \\ L: Line set \\ $S_{p}^{l}$ : observability function at bus $\mathrm{p}$ for $l^{\text {th }}$ line outage
}

\section{References}

Abur A and Magnago F. H., 1999, Optimal meter placement for maintaining observability during single branch outage," IEEE Transactions on Power Systems, Vol. 14, No. 4, pp. 1273-1278.

Ahmadi A., Alinejad-Beromi Y. and Moradi M., 2011, Optimal PMU placement for power system observability using binary particle swarm optimization and considering measurement redundancy, Expert System Applications, vol.38, 7263-7269.

Babu, N. P., Babu, P. S., \& SivaSarma, D. V. S. S., 2015, A wide-area prospective on power system protection: A state-of-art, International Conference on Energy, Power and Environment: Towards Sustainable Growth (ICEPE), IEEE, 1-6.

Baldwin, T.L., Mili, L., Boisen, M.B., Adapa, R., 1993, Power system observability with minimal phasor measurement placement, IEEE Transactions on Power Systems, Vol. 8, No. 2, 707-715.

Babu, N.V.P, Babu P.S., and Sivasarma D. V. S. S., 2015, A Reliable wide-area measurement system using hybrid genetic particle swarm optimization (HGPSO)", International Review of Electrical Engineering, Vol. 10, no. 6, 747-763.

Chakrabarti S. and Kyriakides E., 2008, Optimal placement of phasor measurement units for power system observability, IEEE Transactions on Power Systems, Vol. 23, No. 3, 1433-1440.

Chakrabarti S., Kyriakides E., and Eliades D. G., 2009, Placement of synchronized measurements for power system observability, IEEE Transactions on Power Delivery, vol. 24, no. 1, 12-19.

Caro, E., Singh, R., Pal, B.C., Conejo, A.J., Jabr, R.A. 2012, Participation factor approach for phasor measurement unit placement in power system state estimation, IET Generation Transmission Distribution, vol. 6, no. 9, 922-929.

Gou, B., 2008, Optimal placement of PMUs by integer linear programming", IEEE Transactions on Power Systems, 2008, Vol. 23, No. 3, pp. 1525-1526.

Gherboudj, A., Layeb, A., \& Chikhi, S., 2012, Solving 0-1 knapsack problems by a discrete binary version of cuckoo search algorithm, International Journal of Bio-Inspired Computation, vol. 4, no. 4, 229-236.

Gopakumar P, Chandra G S, Reddy M J B, Mohanta D K., 2013a, Optimal placement of PMUs for the smart grid implementation in Indian power grid - A case study, Frontiers in Energy, Vol. 7, No. 3, 358-372. 
Gopakumar P., Chandra G.S., Jaya M, Reddy B, Mohanta DK, 2013b, Optimal redundant placement of PMUs in Indian power grid - northern, eastern and north-eastern regions, Frontiers in Energy, vol. 7, no. 4, 413-428.

Hajian, M., Ranjbar, A.M., Amraee, T., Shirani, A.R., 2007, Optimal placement of phasor measurement units: particle swarm optimization approach, Proceedings: International Conference on Intelligent Systems Application Power Systems, 1-6.

Kavasseri R., and Srinivasan S. K., 2011, Joint placement of phasor and power flow measurements for observability of power systems, IEEE Transactions on Power Systems, Vol.26, 1929-1936.

Kumar R, Giesselmann MG, Bayne S, and He M, 2014, Generalized method for formulation optimal PMU placement problem, International Journal of Power and Energy Systems, Vol. 34, No. 4, 1-9.

Kulanthaisamy A, Vairamani R, Karunamurthi NK, Koodalsamy C, 2014, Multi-objective PMU placement method considering observability and measurement redundancy using $\mathrm{ABC}$ algorithm, Advances in Electrical and Computer Engineering, Vol. 14, No. 2, pp. $117-128$.

Magnago F. H. and Abur A., 2000, A unified approach to robust meter placement against loss of measurements and branch outages, IEEE Transactions on Power Systems, vol. 15, no. 3, 945-949.

Marın, F.J., Garcı-Lagos, F., Joya, G., Sandoval, F., 2003, Genetic algorithms for optimal placement of phasor measurement units in electric networks', Electronic Letters, vol. 39, no. 19, pp. 1403-1405.

Nazari-Heris, M., Mohammadi-Ivatloo, B., 2015, Optimal placement of phasor measurement units to attain power system observability utilizing an upgraded binary harmony search algorithm, Energy Systems, vol. 6, no. 2, 201-220.

Ouaarab A, Ahiod B, Yang X-S, 2014, Discrete cuckoo search algorithm for the travelling salesman problem, Neural Computing \& Applications, vol. 24, 1659-1669.

Power Grid 2018, Power Grid Corporation of India, www.powergridindia.com, Accessed 12 April 2018.

Pal A, Sanchez G.A., Centeno V.A., Thorp J.S., 2014, A PMU placement scheme ensuring real-time monitoring of critical buses of the network, IEEE Transactions on Power Delivery, vol. 29, no. 2, 510-517.

Rakpenthai C., Premrudeepreechacharn S., Uatrongjit S., Watson N. R. 2007, An optimal PMU placement method against measurement loss and branch outage", IEEE Transactions on Power Delivery, vol. 22, no. 1, pp.101-107, Jan. 2007.

Roy B.K.S., Sinha A.K., Pradhan A.K., 2012. An optimal PMU placement technique for power system observability, Electrical Power and Energy Systems, 42, 71-77.

Srivastava A, Maheswarapu S, 2015, Optimal PMU placement for complete power system observability using binary cat swarm optimization, International Conference on Energy Economics and Environment, Noida, India, 1-6.

Sodhi, R., Srivastava, S.C., Singh, S.N., 2009, Optimal PMU placement to ensure system observability under contingencies, Proceedings Power and Energy Society General Meeting, pp. 1-6.

Singh, B., Sharma, N. K., Tiwari, A. N., Verma, K. S., \& Singh, S. N. 2011, Applications of phasor measurement units (PMUs) in electric power system networks incorporated with FACTS controllers, International Journal of Engineering, Science and Technology, Vol. 3, No. 3, 64-82.

Xu, B., Abur, A. 2004, Observability analysis and measurement placement for system with PMUs, Proceedings of the IEEE Power Systems Conference Exposition, Vol. 2, 943-946.

Yang X-S, 2009a, Nature-Inspired Optimization Algorithms.

Yang X-S 2009b_(auth.),_Xin-She_Yang_(eds.)_Cuckoo

Yang, X-S. and Deb, S., 2010, Engineering optimisation by cuckoo search, International Journal of Mathematical Modelling and Numerical Optimisation, Vol. 1, No. 4, 330-34.

\section{Biographical notes}

N. V. Phanendra Babu, P. Suresh Babu, D.V. S. S. Sivasarma are all of the Department of Electrical Engineering, National Institute of Technology Warangal, India.

Received November 2017

Accepted February 2018

Final acceptance in revised form April 2018 